\title{
ON POWER-BOUNDED OPERATORS AND THE POINTWISE ERGODIC PROPERTY1
}

\author{
MOSHE FEDER
}

\begin{abstract}
We construct a power-bounded isomorphism $T: L_{p} \rightarrow L_{p}$, for $1<p<$
\end{abstract} 2 , such that $\left[n^{-1} \sum_{i=0}^{n-1} T^{i} f\right](t)$ is not a.e. convergent. $T^{-1}$ is also power-bounded.

1. Introduction, definitions and notations. We will say that an operator $T$ : $L_{p}(\Omega, \mathscr{B}, \mu) \rightarrow L_{p}(\Omega, \mathscr{B}, \mu)$ has the pointwise ergodic property (p.e.p.) if for every $f \in L_{p}(\Omega, \mathscr{B}, \mu),\left[n^{-1} \sum_{i=0}^{n-1} T^{i} f\right](t)$ is a.e. convergent in $\Omega$. It is known that $T$ has the p.e.p. in the following cases: for $1<p<\infty, p \neq 2$ and $T$ an isometry (for an invertible isometry, A. Ionescu-Tulcea [7] and for a general isometry, R. V. Chacon and S. A. McGrath [6]), for $1<p<\infty$ and $T$ a positive contraction (M. A. Akcoglu [1]), and for $1<p<\infty$ and $T$ an isomorphism such that $T$ and $T^{-1}$ are both power-bounded and positive (A. de la Torre [12]). D. L. Burkholder [5] constructed a contraction $T: L_{2} \rightarrow L_{2}$ failing to have the p.e.p. Akcoglu and Sucheston [3] remarked that $T$ can be geometrically dilated to an isometry on an $L_{2}$ space that fails to have the p.e.p.

In this paper we construct for each $1<p<2$ an isomorphism $T_{p}: L_{p} \rightarrow L_{p}$ failing to have the p.e.p. so that $T_{p}$ and $T_{p}^{-1}$ both are power-bounded. Moreover, $T_{p}$ is the "same" operator for all $p \in(1,2]$, i.e. if $p<r<2$ then $T_{p}\left(L_{r}\right) \subset L_{r}$ and $\left.T_{p}\right|_{L_{r}}=T_{r}$. In a remark following the construction we outline a modification of the construction so that we have, in addition, $\left[n^{-1} \sum_{i=0}^{n-1} T_{p}^{i}\right](t)$ a.e. divergent in $[0,1]$, for some $f \in L_{p}$.

An "operator" in this paper is always bounded and linear. Let $X$ be a Banach space. If an operator $P: X \rightarrow X$ is idempotent $\left(P^{2}=P\right)$ then $P$ is said to be a projection and $P(X)$ is said to be complemented in $X . I: X \rightarrow X$ denotes the identity. If $A$ is a subset of $X$ then $\overline{\operatorname{conv}} A$ denotes the closed convex hull of $A$ and $[A]$ its closed linear span. $L_{p}$ is $L_{p}(0,1)$ and the norm of $f \in L_{p}$ will be denoted by $\|f\|_{p}$ whenever $\|f\|$ is ambiguous. $f \in L_{p}$ will be treated as a function (defined a.e.). $\left.f\right|_{B}$ is the restriction of $f$ to $B$. Unless otherwise stated, the scalar field may be the real or complex one. The Hilbert space $l_{2}$ will be denoted sometimes by $l_{2}^{R}$ or $l_{2}^{C}$ to distinguish between the real and complex cases. The unit vector basis $\left\{e_{i}\right\}$ in $l_{2}$ is defined by $e_{i}=(0, \ldots, 0,1,0 \ldots)$, the 1 in the $i$ th slot. A sequence $\left\{x_{i}\right\} \subset X$ is equivalent to the unit vector basis of $l_{2}$ if there is an isomorphism of $l_{2}$ and $\left[x_{i}\right]$

Received by the editors October 30, 1980.

1980 Mathematics Subject Classification. Primary 47A35; Secondary 46E30.

${ }^{1}$ Research supported by NSERC Grant No. A3974. The author is grateful to Professor M. A. Akcoglu and Dr. C.-H. Kan for many very helpful discussions and remarks. 
carrying $e_{i}$ to $x_{i}$ for each $i$. If $a$ is an element of an algebra and $n$ is a positive integer then we put $A_{n}(a)=n^{-1} \sum_{i=0}^{n-1} a^{i}$. An operator $T: X \rightarrow X$ is power-bounded if $\sup _{n}\left\|T^{n}\right\|<\infty$.

It will be convenient to work in $L_{p}(0,2)$ rather than in $L_{p}\left(=L_{p}(0,1)\right)$. When functions $f, g \in L_{p}$ are given we denote by $h=\langle f ; g\rangle$ the function $h \in L_{p}(0,2)$ defined by $h(t)=f(t)$ for $t \in[0,1]$ and $h(t)=g(t-1)$ for $t \in(1,2]$. We also put $(f, g)=\int_{0}^{1} f(t) \overline{g(t)} d t$.

2. Power-bounded operators. M. A. Akcoglu [2] modified an idea of D. L. Burkholder [5] to obtain the following.

LEMMA 1. Let $l_{2}^{C}$ be the complex Hilbert space, and for each $n$ let $R_{n}: l_{2}^{C} \rightarrow l_{2}^{C}$ be the orthogonal projection onto $\left[e_{n+1}, e_{n+2}, \ldots\right]$. Let $\left\{\varepsilon_{n}\right\}$ be a sequence of positive real numbers. Then there exists a surjective isometry $S: l_{2}^{C} \rightarrow l_{2}^{C}$ and there is a sequence $0<n_{1}<n_{2}<\cdots$ of integers so that $\left\|A_{n_{k}}(S)-R_{k}\right\|<\varepsilon_{k}$ for every $k$.

COROLlARY 1. Let $l_{2}^{R}$ be the real Hilbert space, $R_{n}: l_{2}^{R} \rightarrow l_{2}^{R}$ defined similarly and $\left\{\varepsilon_{n}\right\}$ as in Lemma 1. Then there is a surjective isometry $S_{R}: l_{2}^{R} \rightarrow l_{2}^{R}$ and there is a sequence $0<n_{1}<n_{2} \cdots$ of integers so that $\left\|A_{n_{k}}\left(S_{R}\right)-R_{2 k}\right\|<\varepsilon_{k}$ for every $k$.

Proof. Let $A: l_{2}^{C} \rightarrow l_{2}^{R}$ be the real-linear map sending $\left(x_{1}, x_{2}, \ldots\right)$ to $\left(\operatorname{Re} x_{1}, \operatorname{Im} x_{1}, \operatorname{Re} x_{2}, \operatorname{Im} x_{2}, \ldots\right)$. Now put $S_{R}=A S A^{-1}$, the $S$ from Lemma 1 .

REMARK 1. Lemma 1 can be generalized so that it remains true for a Banach space $X$ with a basis $\left\{x_{i}\right\}:$ Let $P_{n}: X \rightarrow X$ be given by $P_{n}\left(\sum \alpha_{i} x_{i}\right)=\sum_{i<n} \alpha_{i} x_{i}$, $P_{0}=0$ and let $\left\{\varepsilon_{n}\right\}$ be as in the lemma. Then

(a) There exist a power-bounded isomorphism $T: X \rightarrow X$ and a sequence $0<n_{1}<n_{2}<\cdots$ of integers so that $\left\|A_{n_{k}}(T)-\left(I-P_{k}\right)\right\|<\varepsilon_{k}$ for every $k$.

(b) If the basis $\left\{x_{n}\right\}$ is unconditional and the scalar field the complex numbers, we can make $T$ and $T^{-1}$ both power-bounded.

SKetch OF THE PROOF. If $\left\{\lambda_{n}\right\}$ is a decreasing (increasing) sequence in $[0,1]$ and $T: X \rightarrow X$ is given by $T\left(\sum \alpha_{i} x_{i}\right)=\sum \lambda_{i} \alpha_{i} x_{i}$, then $T \in \overline{\operatorname{conv}}\left\{P_{n}: n=0,1, \ldots\right\}$ (respectively, $\left.T \in \overline{\operatorname{conv}}\left\{I-P_{n}: n=0,1, \ldots\right\}\right)$. Since the basis constant $K=$ $\sup \left\|P_{n}\right\|$ is always finite, $T$ is a well-defined operator and for every $n,\left\|T^{n}\right\|<K$ (respectively, $\left\|T^{n}\right\| \leqslant K+1$ ). Now construct monotone sequences $0<\lambda_{1}<\lambda_{2}$ $<\cdots<1$ and $0<n_{1}<n_{2}<\cdots$ inductively, in a similar way to Akcoglu's construction. Note that if, for a given $j, A_{n}\left(\lambda_{i}\right)<\delta$ for $i<j$ and $A_{n}\left(\lambda_{i}\right)>1-\delta$ for $i \geqslant j+1$ then $A_{n}(T)-\left(I-P_{j}\right)$ is in the set

$$
\delta \cdot \operatorname{conv}\left\{P_{0}, \ldots, P_{j}\right\}+\delta \cdot \overline{\operatorname{conv}}\left\{I-P_{i}: i>j\right\}
$$

and thus of norm less than $(2 K+1) \delta$.

Part (b) is proved in the same way as Akcoglu's lemma (with the $\lambda_{i}$ 's complex, $\left.\left|\lambda_{i}\right|=1\right)$.

LEMMA 2. Let $A_{n}: L_{p} \rightarrow L_{p}$ be a sequence of operators with $\Sigma\left\|A_{n}\right\|<\infty$. Then for every $f \in L_{p}$ the series $\Sigma\left(A_{n} f\right)(t)$ converges a.e. in $[0,1]$.

Proof. Follows easily from

$$
\sum \int_{0}^{1}\left|\left(A_{n} f\right)(t)\right|^{p} d t=\sum\left\|A_{n} f\right\|^{p} \leqslant\|f\|^{p} \sum\left\|A_{n}\right\|^{p}<\infty .
$$


3. Construction of the operator. Let $\left\{r_{n}(t)\right\}$ be the Rademacher functions on $[0,1]$ defined by $r_{n}(t)=\operatorname{sign} \sin 2^{n} \pi t(n=0,1, \ldots)$ where sign $0=1$. The Rademacher functions span a complemented subspace of $L_{p}$ for every $1<p<\infty$, and the sequence $\left\{r_{n}\right\} \in L_{p}$ is equivalent to the unit vector basis of $l_{2}$ for every $1<p<$ $\infty$; i.e. there are positive constants $A_{p}$ and $B_{p}$ so that for every $\left\{a_{n}\right\} \in l_{2}$,

$$
A_{p}\left(\sum\left|a_{n}\right|^{2}\right)^{1 / 2} \leqslant\left\|\sum a_{n} r_{n}\right\|_{p} \leqslant B_{p}\left(\sum\left|a_{n}\right|^{2}\right)^{1 / 2}
$$

(Khinchin's inequality), and the map $V: L_{p} \rightarrow L_{p}$, defined by $V f=\Sigma\left(f, r_{n}\right) r_{n}$ where $\left(f, r_{m}\right)=\int_{0}^{1} f(t) r_{n}(t) d t$, is a (bounded) projection when $1<p<\infty$ (see A. Pełczyński [11], Proposition 5). D. Menchoff [10] constructed an orthonormal sequence $\left\{g_{n}\right\}$ in $L_{2}$ and a sequence of real numbers $\left\{c_{n}\right\} \in l_{2}$ so that $\sum c_{n} g_{n}(t)$ is a.e. divergent in $[0,1]$. Define $\left\{\rho_{n}\right\}$ and $\left\{y_{n}\right\}$ in $L_{p}(0,2)$ by $\rho_{n}=\left\langle r_{n} ; 0\right\rangle, y_{2 n-1}=$ $\left\langle 0 ; g_{n}\right\rangle$ and $y_{2 n}=0$. Let $\left\{a_{n}\right\} \in l_{2}$. Then for $p \in(1,2]$,

$$
\begin{aligned}
A_{p}\left(\sum\left|a_{n}\right|^{2}\right)^{1 / 2} & \leqslant\left\|\sum a_{n} r_{n}\right\|_{p} \leqslant\left\|\sum a_{n}\left(\rho_{n}+y_{n}\right)\right\|_{p} \\
& \leqslant\left\|\sum a_{n} \rho_{n}\right\|_{p}+\left\|\sum a_{n} y_{n}\right\|_{p} \\
& \leqslant\left\|\sum a_{n} r_{n}\right\|_{2}+\left\|\sum a_{2 n-1} g_{n}\right\|_{2} \leqslant 2\left(\sum\left|a_{n}\right|^{2}\right)^{1 / 2} .
\end{aligned}
$$

Thus $B e_{i}=\rho_{i}+y_{i}$ defines an isomorphism $B: l_{2} \rightarrow\left[\rho_{n}+y_{n}\right]$. Let $R: L_{p}(0,2) \rightarrow L_{p}$ be the restriction map $R f=\left.f\right|_{[0,1]}$ and let $A:\left[r_{n}\right] \rightarrow L_{p}(0,2)$ be given by $A\left(r_{n}\right)=\rho_{n}$ $+y_{n} \cdot A$ is an isomorphism (into). We put $U=A V R$. For every $n$,

$$
U\left(\rho_{n}+y_{n}\right)=A V R\left(\rho_{n}+y_{n}\right)=A V\left(r_{n}\right)=A\left(r_{n}\right)=\rho_{n}+y_{n} .
$$

Therefore $U$ is a projection of $L_{p}(0,2)$ onto $\left[\rho_{n}+y_{n}\right]$. For a.e. $t \in[1,2]$ the series $\sum c_{n}\left(\rho_{2 n-1}+y_{2 n-1}\right)(t)=\sum c_{n} g_{n}(t-1)$ is divergent. Denote

$$
f=\sum c_{n}\left(\rho_{2 n-1}+y_{2 n-1}\right) \in L_{p}(0,2) \text {. }
$$

By Lemma 1 and Corollary 2 , there is an isometry $S: l_{2} \rightarrow l_{2}$ and a sequence $0<n_{1}<n_{2}<\cdots$ of integers so that $\Sigma\left\|A_{n_{k}}(S)-R_{2 k}\right\|<\infty$. Now put $T=$ $B S B^{-1} U+I-U . T$ is an isomorphism of $L_{p}(0,2), T$ and $T^{-1}$ both are powerbounded, and $\left[A_{n}(T) f\right](t)$ is a.e. divergent in $[1,2]$ :

$$
\left[B R_{2 k} B^{-1} U f\right](t)=\sum_{i=k+1}^{\infty} c_{i}\left(\rho_{2 i-1}+y_{2 i-1}\right)(t)
$$

for every $t$ and

$$
\sum\left\|A_{n_{k}}(T)-\left(B R_{2 k} B^{-1} U+I-U\right)\right\|<\infty .
$$

Since $(I-U) f=0$, we have the result using Lemma 2 .

REMARK 2. A technically more involved construction gives, for $1<p \leqslant 2$, an isomorphism $T: L_{p}(0,2) \rightarrow L_{p}(0,2)$ having, in addition, the property that $\left[A_{n}(T) f\right](t)$ is divergent a.e. in $[0,2]$.

SKetch of THE Construction. Since $p>1$, the Haar system $\left\{\phi_{i}\right\}$ is an unconditional basis of $L_{p}$ (see, e.g., [9]). A simple modification of Kaczmarz' version [8] of Menchoff's construction gives an orthonormal sequence $\left\{g_{i}\right\}$ in $L_{2}$ and a sequence $\left\{c_{i}\right\} \in l_{2}$ with $\Sigma c_{i} g_{i}(t)$ a.e. divergent in $[0,1]$ such that there are sequences of 
integers $0<k_{1}<k_{2}<\cdots$ and $0<m_{1}<m_{2}<\cdots$ satisfying for every $i, g_{i} \in$ $\left[\phi_{k_{2 i}+1}, \ldots, \phi_{k_{2 i}}\right]$ and $r_{m_{i}} \in\left[\phi_{k_{2 i+1}+1}, \ldots, \phi_{k_{2 i+2}}\right]$. Now define a sequence $\left\{y_{i}\right\}$ in $L_{p}(0,2)$ by

$$
\begin{gathered}
y_{4 i-3}=\left\langle r_{m_{2 i-1}} ; g_{i}\right\rangle, \quad y_{4 i-2}=\left\langle r_{m_{2 i}} ; 0\right\rangle, \\
y_{4 i-1}=\left\langle g_{i} ; r_{m_{2 i-1}}\right\rangle, \quad y_{4 i}=\left\langle 0 ; r_{m_{2 i}}\right\rangle .
\end{gathered}
$$

Let $\left\{a_{i}\right\} \in l_{2}$. Since $1<p \leqslant 2$,

$$
\begin{aligned}
\left\|\sum a_{i} y_{i}\right\| & \geqslant\left\|\left.\sum a_{i} y_{i}\right|_{[0,1]}\right\| \geqslant K_{p}\left\|\sum\left(a_{4 i-3} r_{m_{2 i-1}}+a_{4 i-2} r_{m_{2 i}}\right)\right\| \\
& \geqslant K_{p} A_{p}\left[\sum\left(\left[a_{r 4 i-3}\right]^{2}+\left[a_{s 4 i-1}\right]^{2}\right)\right]^{1 / 2}
\end{aligned}
$$

where $K_{p}$ is the unconditional constant of the Haar basis. Similarly,

$$
\left\|\sum a_{i} y_{i}\right\| \geqslant K_{p} A_{p}\left[\sum\left(\left|a_{4 i-1}\right|^{2}+\left|a_{4 i}\right|^{2}\right)\right]^{1 / 2} \text {. }
$$

Thus

$$
\left\|\sum a_{i} y_{i}\right\| \geqslant 2^{-1 / 2} K_{p} A_{p}\left(\sum\left|a_{i}\right|^{2}\right)^{1 / 2} \text {. }
$$

It is easy to see that $\left\|\sum a_{i} y_{i}\right\| \leqslant 2^{1 / \mathrm{p}}\left(\sum\left|a_{i}\right|^{2}\right)^{1 / 2}$. Thus, $\left\{y_{i}\right\}$ is equivalent to the unit vector basis of $l_{2}$. Now define $R_{j}: L_{p}(0,2) \rightarrow L_{p}(j=1,2)$ by $R_{1} f=\left.f\right|_{[0,1]}$ and $\left[R_{2} f\right](t)=f(t+1)$ for $t \in[0,1]$. Define $A_{j}:\left[r_{m_{i}}\right] \rightarrow\left[y_{i}\right](j=1,2)$ by

$$
\begin{array}{ll}
A_{1} r_{m_{2 i-1}}=y_{4 i-3}, & A_{1} r_{m_{2 i}}=y_{4 i-2}, \\
A_{2} r_{m_{2 i-1}}=y_{4 i-1}, & A_{2} r_{m_{2 i}}=y_{4 i} .
\end{array}
$$

Let $V$ be the projection in $L_{p}$ defined by $V f=\Sigma\left(f, r_{m_{i}}\right) r_{m_{i}}$. The map $P: L_{p}(0,2) \rightarrow$ $L_{p}(0,2), P=A_{1} V R_{1}+A_{2} V R_{2}$ is a projection onto [ $\left.y_{i}\right]$ (note that $V g_{i}=0$ for all $i$ ). Put $f=\sum c_{i}\left(y_{4 i-3}+y_{4 i-1}\right)$; then $\sum c_{i}\left(y_{4 i-3}+y_{4 i-1}\right)(t)$ is a.e. divergent in [0,2] (to see this note that $\sum c_{i} r_{m_{2 i-1}}(t)$ is a.e. convergent in [0, 1]-cf. [4]). The end of the construction is similar to the preceding one.

REMarK 3. The Haar basis $\left\{\phi_{i}\right\}$ has the following property: if $\Sigma \alpha_{i} \phi_{i} \in L_{p}$ then $\sum \alpha_{i} \phi_{i}(t)$ converges a.e. in [0,1] (see [4]). Using a similar idea to the one used in our construction we can construct an unconditional basis $\left\{x_{i}\right\}$ of $L_{p}$ and a function $f=\sum \alpha_{i} x_{i} \in L_{p}$ with $\sum \alpha_{i} x_{i}(t)$ a.e. divergent in [0,1]. Again, for reasons of convenience we consider $L_{p}(0,2)$ instead of $L_{p}$. Using Pelczyński's decomposition method ([11]; see also [9]), it is easy to see that if $P$ is as in Remark 2 then $(I-P) L_{p}(0,2)$ is isomorphic to $L_{p}$ and thus has an unconditional basis $\left\{z_{i}\right\}$. Now define $x_{2 i-1}=y_{i}, x_{2 i}=z_{i}$ and $f$ as in Remark 2 .

\section{REFERENCES}

1. M. A. Akcoglu, A pointwise ergodic theorem in $L_{p}$-spaces, Canad. J. Math. 27 (1975), 1075-1082.

2. ___ Pointwise ergodic theorems in $L_{p}$ spaces, (Proc. Conf. Ergodic Theory, Oberwolfach, 1978), Lecture Notes in Math., vol. 729, Springer-Verlag, Berlin and New York, 1979, pp. 13-15.

3. M. A. Akcoglu and L. Sucheston, Remarks on dilations in $L_{p}$-spaces, Proc. Amer. Math. Soc. 53 (1975), 80-82.

4. G. Alexits, Convergence problems of orthogonal series, Pergamon Press, New York, 1961.

5. D. L. Burkholder, Semi-Gaussian subspaces, Trans. Amer. Math. Soc. 104 (1962), 123-131.

6. R. V. Chacon and S. A. McGrath, Estimates of positive contractions, Pacific J. Math. 30 (1969), 609-620. 
7. A. Ionescu-Tulcea, Ergodic properties of isometries in $L_{p}$-spaces, Bull. Amer. Math. Soc. 70 (1964), 366-371.

8. S. Kaczmarz, Notes on orthgonal series. II, Studia Math. 5 (1934), 103-106.

9. J. Lindenstrauss and L. Tzafriri, Classical Banach spaces. I, Springer-Verlag, Berlin and New York, 1977.

10. D. Menchoff, Sur les séries de fonctions orthogonales, Fund. Math. 4 (1923), 82-105.

11. A. Pełczyński, Projections in certain Banach spaces, Studia Math. 19 (1960), 209-228.

12. A. de la Torre, Simple proof of the maximal engodic theorem, Canad. J. Math. 28 (1976), 1073-1075.

Department of Mathematics, University of Toronto, Toronto, Ontario, Canada M5S 1A1

Current address: Department of Mathematics, Texas A \& M University, College Station, Texas 77843 